\title{
HIGH FREQUENCY BEHAVIOR OF TRANSVERSE IMPEDANCE FOR A CAVITY IN A BEAM PIPE*
}

\author{
A.V. Fedotov $^{\dagger}$, R.L. Gluckstern, and M. Venturini \\ Physics Department, University of Maryland, College Park, MD 20742
}

\begin{abstract}
The interest in short bunches in many accelerator applications requires good understanding of the high frequency behavior of the interaction between the beam and its environment. In this paper we report an analytic calculation for the high frequency limit of the transverse impedance for both a single and periodic array of cavities in a beam pipe.
\end{abstract}

\section{INTRODUCTION}

The acceleration of charged particles in periodic structures leads to wakefields which are capable of interacting adversely with particles in the same bunch, or in following bunches. The conventional method of describing these limitations in the current that can be accelerated involves the longitudinal and transverse coupling impedances of the structure. With the increasing use of short bunches, it becomes necessary to evaluate these coupling impedances at high frequencies (wavelength of the order of the bunch length). This has been done for the longitudinal impedance of small periodic obstacles in an azimuthally symmetric structure [1], where we have derived an integral equation for the axial electric field at the inner bore radius. In this paper we address the corresponding problem for the transverse coupling impedance at high frequency. In particular, we first derive the integral equation for the electric field at the bore radius of the structure and obtain the result for the transverse impedance of a single small obstacle at high frequency by way of the high frequency limit of the kernels. The problem is more complicated than it was for the longitudinal impedance, since both TM and TE waveguide modes are present, which requires matching two components of the magnetic field at the boundary between the waveguide and the obstacle. As we shall eventually see, however, in the high frequency limit, the TM contribution dominates for a single obstacle, but the TE contributions must be included in the periodic case. Nevertheless, we find the same relation between the longitudinal and transverse impedances as exists for the resistive wall impedances of a beam pipe.

We now consider a point charge $Q$ traveling in the $z$ direction at $(x, y)=\left(\Delta_{x}, 0\right)$. We start with the definition of the $x$ component of the transverse coupling impedance

* Work supported by the U.S. Department of Energy

† fedotov@physics.umd.edu

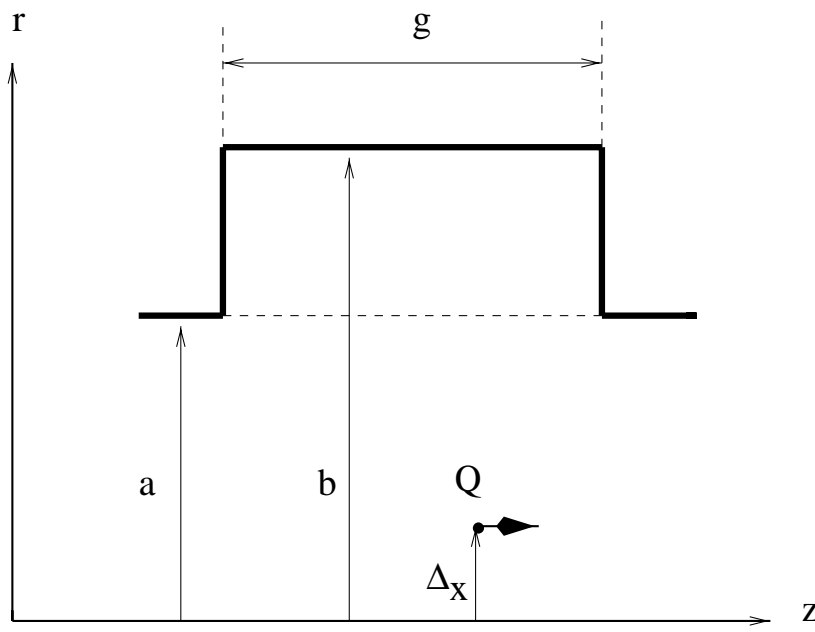

Figure 1: The layout of geometry for a single cavity.

as a function of $k=\omega / c$. In the limit of small $\Delta_{x}$ we have

$$
\begin{aligned}
Z_{x}(k) & =\frac{j}{Q \Delta_{x}} \int_{-\infty}^{\infty} d z e^{j k z}\left[E_{x}(z ; k)\right. \\
& \left.-Z_{0} H_{y}(z ; k)\right]_{\substack{x=0 \\
y=0}}
\end{aligned}
$$

in units of $[\Omega / m]$ (of transverse displacement). Here $Z_{0}=$ $\sqrt{\mu_{0} / \epsilon_{0}}=120 \pi[\Omega]$ is the impedance of free space. We then use

$$
\frac{\partial E_{x}}{\partial z}-\frac{\partial E_{z}}{\partial x}=-j k Z_{0} H_{y}
$$

to obtain the alternate expression [2]:

$$
Z_{x}(k)=-\left.\frac{1}{Q \Delta_{x} k} \int_{-\infty}^{\infty} d z e^{j k z} \frac{\partial E_{z}}{\partial x}\right|_{x=0, y=0},
$$

where we use the time dependence $\exp (j \omega t)$.

The general technique consists of expanding fields in both the pipe $(r \leq a)$ and cavity $(a \leq r \leq b)$ regions into a complete set of functions. At the common interface (shown on Fig. 1) the fields have to be matched, yielding equations for the expansion coefficients.

\section{INTEGRAL EQUATIONS AND SOLUTION FOR IMPEDANCE}

We first consider a single cavity of radius $b$ and axial length $g$. Matching of the azimuthal and longitudinal components 
of the magnetic field at the pipe radius $r=a$ for $0 \leq z \leq g$ (see Fig. 1), leads to the integral equations

$$
\begin{aligned}
1= & \int_{0}^{g} d z^{\prime}\left[\mathcal{P}_{11}\left(z, z^{\prime}\right)+\mathcal{Q}_{11}\left(z, z^{\prime}\right)\right] e_{z}\left(z^{\prime}\right) \\
+ & \int_{0}^{g} d z^{\prime} \mathcal{Q}_{12}\left(z, z^{\prime}\right) e_{\theta}\left(z^{\prime}\right) \\
0 & =\int_{0}^{g} d z^{\prime} \mathcal{Q}_{21}\left(z, z^{\prime}\right) e_{z}\left(z^{\prime}\right) \\
& +\int_{0}^{g} d z^{\prime} \tilde{\mathcal{Q}}_{22}\left(z, z^{\prime}\right) \frac{d e_{\theta}\left(z^{\prime}\right)}{d z^{\prime}}
\end{aligned}
$$

where

$$
\begin{aligned}
& \mathcal{P}\left(z, z^{\prime}\right)=\mathcal{P}^{p}\left(z-z^{\prime}\right)+\mathcal{P}^{c}\left(z, z^{\prime}\right) \\
& \mathcal{Q}\left(z, z^{\prime}\right)=\mathcal{Q}^{p}\left(z-z^{\prime}\right)+\mathcal{Q}^{c}\left(z, z^{\prime}\right)
\end{aligned}
$$

and $\tilde{\mathcal{Q}}_{22}\left(z, z^{\prime}\right)=-\int d z^{\prime} Q_{22}\left(z, z^{\prime}\right)$. In Eqs. (4) and (5) we defined

$$
\begin{aligned}
& E_{z}(z) \equiv \frac{Q \Delta_{x} Z_{0}}{\pi a^{2}} e^{-j k z} e_{z}(z), \\
& E_{\theta}(z) \equiv \frac{Q \Delta_{x} Z_{0}}{\pi a^{2}} e^{-j k z} e_{\theta}(z) .
\end{aligned}
$$

The kernels denoted by $\mathcal{P}(u)$ come from TM modes and those denoted by $\mathcal{Q}(u)$ come from TE modes. We use the superscript $p$ for the pipe kernels and the superscript $c$ for the cavity kernels.

Our next task is to compute the high frequency (large $k$ ) limit for the kernels after averaging over fast oscillations. The details are contained in [3]. All kernels vanish for $u=$ $z-z^{\prime} \leq 0$. For $u>0$ the leading terms in the high $k$ limit are:

$$
\begin{aligned}
\mathcal{P}_{11}^{p}(u) & +\mathcal{Q}_{11}^{p}(u)=\mathcal{P}_{11}^{c}(u)+\mathcal{Q}_{11}^{c}(u) \\
& \cong-(1+j) \sqrt{k /(4 \pi u)}, \\
\mathcal{Q}_{12}^{p}(u) & =\mathcal{Q}_{21}^{p}(u)=\mathcal{Q}_{12}^{c}(u)=\mathcal{Q}_{21}^{c}(u) \\
& \cong(1-j) / \sqrt{4 a^{2} \pi k u}, \\
\tilde{\mathcal{Q}}_{22}^{p}(u) & =\tilde{\mathcal{Q}}_{22}^{c}(u) \cong(1-j) / \sqrt{\pi k u} .
\end{aligned}
$$

Remarkably, the corresponding pipe and cavity kernels are identical for large $k$ after smoothing. The same situation prevailed in our earlier calculation for the smoothed longitudinal coupling impedance at high frequency for a single obstacle with only TM modes being considered [1]. Thus the integral equations become:

$$
\begin{aligned}
1 & =-(1+j) \sqrt{\frac{k}{\pi}} \int_{0}^{z} \frac{d z^{\prime} e_{z}\left(z^{\prime}\right)}{\sqrt{z-z^{\prime}}} \\
& +\frac{1-j}{a \sqrt{\pi k}} \int_{0}^{z} \frac{d z^{\prime} e_{\theta}\left(z^{\prime}\right)}{\sqrt{z-z^{\prime}}}
\end{aligned}
$$

$$
\begin{aligned}
0 & =\frac{1-j}{a \sqrt{\pi k}} \int_{0}^{z} \frac{d z^{\prime} e_{z}\left(z^{\prime}\right)}{\sqrt{z-z^{\prime}}} \\
& +\frac{2(1-j)}{\sqrt{\pi k}} \int_{0}^{z} \frac{d z^{\prime}}{\sqrt{z-z^{\prime}}} \frac{d e_{\theta}}{d z^{\prime}} .
\end{aligned}
$$

From Eq. (14) we see that, for large $k$,

$$
e_{z}\left(z^{\prime}\right)=-2 a \frac{d e_{\theta}}{d z^{\prime}} .
$$

The term involving $e_{\theta}\left(z^{\prime}\right)$ in Eq. (13) is therefore of order $1 /(k a)$ compared with the term in $e_{z}\left(z^{\prime}\right)$ and can consequently be neglected. The solution for $e_{z}\left(z^{\prime}\right)$ is then [4]:

$$
e_{z}\left(z^{\prime}\right) \cong-\frac{(1-j)}{2 \sqrt{\pi k z^{\prime}}}
$$

In order to obtain the impedance, defined in Eq. (3), we use the equation for the axial electric field in the pipe region

$$
E_{z}(r, z ; k)=\int_{-\infty}^{\infty} d q A(q) \frac{J_{1}(\kappa r)}{J_{1}(\kappa a)} e^{-j q z}
$$

to show that

$$
\left.\frac{\partial E_{z}}{\partial x}\right|_{r=0}=\int_{-\infty}^{\infty} \frac{\kappa}{2} \frac{d q A(q)}{J_{1}(\kappa a)} e^{-j q z} .
$$

Here $\kappa^{2} \equiv k^{2}-q^{2}$ and the contour in the complex $q$ plane goes below the poles at $J_{1}(\kappa a)=0$ (TM propagating modes) on the negative $q$ axis and above the poles on the positive $q$ axis. Equation (3) then becomes

$$
Z_{x}(k)=-\frac{q}{Q \Delta_{x} k} \int_{-\infty}^{\infty} \frac{\kappa d q A(q)}{2 J_{1}(\kappa a)} \int_{-\infty}^{\infty} d z e^{j(k-q) z} .
$$

The integral over $z$ in Eq. (19) is $2 \pi \delta(q-k)$, leading to

$$
Z_{x}(k)=-\frac{2 \pi A(k)}{Q \Delta_{x} k a}=\frac{-1}{Q \Delta_{x} k a} \int_{0}^{g} d z E_{z}(z) e^{j k z} .
$$

Finally, by using Eqs. (8) and (16) in Eq. (20), we find

$$
\frac{Z_{x}(k)}{Z_{0}}=\frac{-1}{\pi k a^{3}} \int_{0}^{g} d z^{\prime} e_{z}\left(z^{\prime}\right) \cong \frac{(1-j)}{a^{3}} \sqrt{\frac{g}{\pi^{3} k^{3}}} .
$$

We note that the high frequency dependence of the transverse impedance for a single cavity arises essentially from the TM cavity and pipe kernels. Furthermore, we see that

$$
\frac{Z_{x}(k)}{Z_{0}} \cong \frac{2}{k a^{2}} \frac{Z_{\|}(k)}{Z_{0}},
$$

where $Z_{\|}(k)$ is the high frequency limit for the longitudinal impedance of a single cavity [1], a relation which also applies to a lossy beam pipe [5]. In fact, in the high-frequency limit for a single cavity, simple arguments of diffraction theory can be used to obtain the same factor $2 /\left(k a^{2}\right)$ as in Eq. (22) [6]. Here we used a more rigorous treatment and showed that TE modes give only next order frequency corrections, ensuring that only TM modes will govern the high-frequency behavior of the transverse impedance of a single cavity and providing the factor $2 /\left(k a^{2}\right)$. 


\section{PERIODIC ARRAY OF CAVITIES}

We are now ready to extend the calculation of the transverse impedance to a periodic array of identical cavities. In the geometry of the system considered, $g$ is the length of each cavity and $L$ is the axial distance (center to center) between two adjacent cavities.

We obtain the integral equations satisfied by the fields using the same steps as we followed for the case of a single cavity. The only difference is that in doing the field matching in each cavity one has to take into account the contributions to the fields coming from all the other cavities. These contributions determine the appearence of the coupling kernels in the integral equations. As for the case of a single cavity we find that in the high frequency limit we can reduce the original system of two integral equations to a single equation for the unknown axial component of the electric field $e_{z}$. This integral equation has the same form as the one in the calculation of the longitudinal impedance [1]. Therefore, for the transverse impedance of a periodic structure at high frequency we finally obtain [3]:

$$
Z_{x}(g / L)=\frac{2}{k a^{2}} Z_{\|}(g / L) .
$$

The problem is then reduced to calculating $Z_{\|}$from the solution of the integral equation for $e_{z}$. Although it was not explicitly stated in the paper, the final form for the integral equation in [1] was correct only in the limit $g / L \ll 1$, because of the way the coupling kernels were approximated. Recently, Yokoya [7] pointed out that one can treat the case of a general $g / L$ by retaining an extra term of order $k^{-1 / 2}$ compared with the coupling kernel [1]. The resulting integral equation can be approximately solved [3], [7] with the following result for the impedance per period $Z_{\|}(g / L)$ :

$$
\frac{Z_{\|}}{Z_{0}}=\frac{-j L}{\pi k a^{2}}\left[1+(1-j) \frac{L}{a} \sqrt{\frac{\pi}{k g}} \lambda(g / L)\right]^{-1},
$$

with

$$
\begin{aligned}
\lambda(\xi) \cong 1+\frac{\alpha_{0}}{\pi} \xi^{1 / 2}-\frac{\alpha_{1}}{3 \pi} \xi^{3 / 2}+\frac{13 \alpha_{2}}{45 \pi} \xi^{5 / 2} \\
+\frac{8 \alpha_{1}^{2}}{45 \pi^{2}} \xi^{3}-O\left(\xi^{7 / 2}\right)
\end{aligned}
$$

where $\alpha_{0}=\zeta(1 / 2), \alpha_{1}=\zeta(3 / 2) / 2, \alpha_{2}=3 \zeta(5 / 2) / 8$, and $\zeta$ denotes the Riemann zeta function. In the limit $g \ll$ $L$ we have $\lambda=1$, and one recovers our earlier expression for the longitudinal impedance [1]. In the case $g / L=1$ it is possible to obtain an exact analytic result, using diffraction theory, as was shown by Stupakov [8]. In our notation Stupakov's result is $\lambda(1)=-\zeta(1 / 2) / \pi \cong 0.464845$ [8], in agreement with Yokoya's numerical solution [7].

Equations (23)-(25) provide an analytic description of the transverse and longitudinal impedance at high frequency for an infinite array of periodic cavities. These expressions are valid when $N L \gg k a^{2}$, where $N$ is the number of cavities. For $\mathrm{ka}^{2} / L \geq N \gg 1$ there is a transition to the result valid for a finite number of cavities, and one needs to use a different expression for the longitudinal impedance [9].

\section{SUMMARY}

In this paper we address the question of the transverse coupling impedance at high frequency. The problem is more complicated than for the longitudinal impedance, since both TM and TE waveguide modes are present, which requires matching two components of the magnetic field at the boundary between the waveguide and the obstacle. We show that, in the high frequency limit, the TM contribution dominates for a single obstacle, while the TE contribution must be included in the periodic case. Nevertheless, we find the same relation between the longitudinal and transverse impedances as exists for the resistive wall impedance of a beam pipe. Validity of the factor $2 /\left(k a^{2}\right)$ between the longitudinal and transverse impedances allows simple estimates of the transverse impedance at high frequency.

\section{ACKNOWLEDGMENT}

We are grateful to K. Yokoya for sharing his notes with us and for making several useful comments. We also wish to thank S. Kurennoy for helpful comments.

\section{REFERENCES}

[1] R.L.Gluckstern, Phys. Rev. D 39, 2773 (1989); ibid., 2780.

[2] See, for example, R.L.Gluckstern, Phys. Rev. A 46, 1106 (1992).

[3] A.V. Fedotov, R.L. Gluckstern and M. Venturini, Transverse Impedance of a Periodic Array of Cavities, submitted for publication in Phys. Rev. ST Accel. Beams, 1999.

[4] We note at this point, that the behavior of $e_{z}\left(z^{\prime}\right)$ near $z^{\prime}=0$ is expected to be $\left(k z^{\prime}\right)^{-1 / 3}$ for $k z^{\prime} \ll 1\left(z^{\prime}\right.$ small compared with the wavlength $2 \pi / k$ ). Thus $\left(k z^{\prime}\right)^{1 / 2}$ in the denominator of Eq. (16) should be replaced by $\left(k z^{\prime}\right)^{1 / 2}+$ const $\left(k z^{\prime}\right)^{1 / 3}$, where const is $\mathcal{O}(1)$. Nevertheless, the dominant region for the integration over $z^{\prime}$ in Eq. (21) is for $k z^{\prime} \sim k g \gg 1$, and thus $\left(k z^{\prime}\right)^{1 / 2} \gg\left(k z^{\prime}\right)^{1 / 3}$. It is for this reason that Eq. (16) does not accurately reflect the behavior for values of $k z^{\prime} \ll$ 1, but this has no effect on the final result in Eq. (21).

[5] A. Chao, Physics of collective beam instabilities in high energy accelerators (Wiley, New York, 1993).

[6] K. Bane and M. Sands, Part. Accel., 25, 73 (1990).

[7] K. Yokoya, private communication; also K.L.F. Bane and K. Yokoya, The High-Frequency Impedance of a Periodic Accelerator Structure, these Proceedings.

[8] G. Stupakov, In Proceedings of the 1995 Partical Accelerator Conference, Dallas, TX (IEEE, Piscataway, NJ, 1996), p. 3303.

[9] R.L. Gluckstern, In Proceedings of the 1989 Particle Accelerator Conference, Chicago, IL (IEEE, Piscataway, NJ, 1989), p. 1157. 